\title{
Pancreatic transplantation with delayed duct occlusion versus bladder drainage: long-term results
}

\author{
A. Königsrainer, W. Steurer, C. Aichberger, R. Gassner, Th. Schmid and R. Margreiter \\ Department of Transplant Surgery, University Hospital, Innsbruck, Austria
}

Summary. Between April 1985 and August 1990 a total of 51 combined pancreas kidney transplants and 6 single pancreas transplants were performed in 51 Type 1 (insulin - dependent) diabetic patients suffering from end-stage diabetic nephropathy and three patients with proliferative retinopathy. In 17 transplants the pancreatic duct was occluded with a mean delay of 53 days (Group 1). Because of a high incidence of local complications associated with a prolonged hospitalization this technique was abandoned despite favourable results: The actual survival rates for patients, pancreas and renal allografts at 1 year are $94 \%, 72 \%$ and $93 \%$, respectively. From 1987 a total of 39 consecutive segmental pancreas grafts were anastomosed with the urinary bladder (Group 2). Pancreatic secretions were temporarily drained to the exterior in all patients via a duct catheter. Monitoring of the exocrine function including pancreatic secretion cytology and pancreatic secretion neopterin excretion proved to be reliable rejection markers. Survival rates at 1 year were calculated to be $90 \%, 74 \%$ and $89 \%$ for all patients, pancreas grafts and renal grafts. Apart from local complications in group I which did not cause any graft loss, the surgical complication rate was comparably low in both groups.

Key words: Pancreatic transplantation - Delayed duct occlusion - Bladder drainage - Long- term results

\section{Introduction}

The problem of how to handle exocrine pancreas secretion after pancreas transplantation remains unsolved. Occlusion of the pancreatic duct with neoprene or prolamine at the time of transplantation (Dubernard et al. 1978) or delayed occlusion after temporary drainage (Königsrainer et al. 1987) represent the safest surgical techniques. Apart from local complications such as formation of fistulas and pseudocysts, progressive fibrosis eventually leading to loss of endocrine function must be considered as the major drawback of this technique (Margreiter et al. 1988). In an attempt to preserve the anatomical structure of the graft, most centres now prefer drainage of the exocrine secretion either into the bladder or the gut (Sutherland et al. 1989). The advantage of unphysiological drainage into the lower urinary tract over physiological diversion into the gut is the simplicity of the surgical procedure together with the possibility of monitoring the graft by measuring pancreas-specific enzymes in the urine (Sollinger et al. 1985). Although good results were achieved with the delayed duct occlusion technique, we have abandoned this method in favour of bladder drainage. Bladder drainage, despite its high incidence of local complications, does not lead to loss graft function but is associated with prolonged hospitalization (Margreiter et al. 1989).

\section{Subjects and methods}

Between April 1985 and August 1990 a total of 57 pancreas transplantations were performed at our institution in 54 Type 1 diabetic patients. Because of end-stage diabetic nephropathy, 51 patients were also given a renal transplant from the same donor. In this study the patients were divided into two groups (Table 1) according to the type of pancreatic duct drainage used.

Table 1. Characteristics of patients before pancreatic transplantation

\begin{tabular}{lcc}
\hline & $\begin{array}{c}\text { Delayed duct } \\
\text { occlusion } \\
\text { group }\end{array}$ & $\begin{array}{c}\text { Bladder } \\
\text { drainage } \\
\text { group }\end{array}$ \\
\hline Patients (n) & 16 & 38 \\
Age (years) & $36.3(22-55)$ & $36.9(19-59)$ \\
Sex (male) & 8 & 25 \\
(f emale) & 7 & 13 \\
Duration of diabetes (years) & $20.5(13-31)$ & $23.0(15-37)$ \\
Time on dialysis (months) & $26.0(0-156)$ & $26.5(0-110)$ \\
HLA mismatches AB & $2.7(1-4)$ & $3.1(2-4)$ \\
& $1.5(0-2)$ & $1.5(1-2)$ \\
SKP (n) DR & 15 & 34 \\
PA (n) & 1 & 4 \\
2nd transplantation $(n)$ & 1 & 1 \\
\hline
\end{tabular}

SKP: simultaneous kidney-pancreas transplantation; PA: pancreas alone; 
The pancreas grafts consisted not only of the tail but also of parts of the head of the gland. Graft vessels were anastomosed to the exterior iliac vessels in an end-side fashion. For arterial anastomosis the coeliac axis or, in case of liver procurement, the splenic artery extended with an iliac autograft and the portal vein were used. For rheological reasons the superior mesenteric vein was later used for venous drainage instead of the portal vein (Königsrainer et al. 1990a). Great care was taken to provide an adequate vascular pedicle as well as an obtuse angle between splenic vein and portal vein or superior mesenteric vein. In all instances the graft was placed intraperitoneally. The kidney was transplanted to the left iliac fossa according to standardized techniques. Until the end of 1986 the raw surface of the pancreas was oversown and extraperitonealized in the right lower abdomen. The pancreatic secretions were drained to the exterior by means of a drain inserted into the pancreatic duct. After stabilization of graft function which occurred after a mean of 53 (4072) days, the pancreatic duct was occluded with an alcoholic prolamine solution. In the subsequent 39 pancreas transplants the transected surface of the graft was anastomosed with the bladder in two layers. Pancreatic secretions were also temporarily drained to the exterior with a duct catheter brought out through the bladder and abdominal wall. This drain was pulled out after $30(6-48)$ days. In order to prevent vascular thrombosis, all patients were given low molecular weight dextrans peri-operatively and heparin between days 1 and 10. Thereafter, patients received anti-aggregation medication for 6 months. Prophylactic immunosuppression consisted of cyclosporin plus steroids and azathioprine. Acute rejection episodes were treated with $500 \mathrm{mg}$ methylprednisolone on three consecutive days. In case of further acute rejection or steroid-resistant acute rejection, Antithymocyte globulin was given for 10 days at a dosage of $5 \mathrm{mg} / \mathrm{kg}$ body weight. Various exocrine and endocrine parameters were measured for graft monitoring (Table 2) (Steiner et al. 1987). In particular, pancreatic secretion cytology (Klima et al. 1989) and pancreatic secretion neopterin (Königsrainer et al. 1990b) excretion proved useful in diagnosing acute rejection. Informed consent was obtained from all patients and surgical technique approved by the local ethical committee.

Table 2. Graft monitoring after pancreas transplantation

\section{Exocrine graft function}

in hospital : pancreatic secretion quantity amylase activity cytology

long - term : urinary amylase activity neopterin

Endocrine graft function

in hospital : blood sugar 6 times per day

$$
\begin{aligned}
& \text { long - term : HbAlc } \text { OGIT } \\
& \text { C - peptide }
\end{aligned}
$$

12 times per year 4 times per year 4 times per year

Renal graft function daily

Renal graft histology

as necessary

Urinary neopterin excretion daily

Technetium perfusion scan as necessary

Biopsy ( cystoscopy )

as necessary

Open biopsy

as necessary

\section{Results}

\section{Delayed duct occlusion technique}

A 23-year-old female patient died of extended myocardial reinfarction 2 weeks after transplantation with two normally functioning transplants. Another patient died after amputation of both legs four years following grafting. One recipient irreversibly rejected both grafts and one non-uraemic female patient rejected her single pancreas transplant. Another pancreas graft had to be removed due to severe pancreatitis. Two more grafts were rejected after 10 months and 3 years, respectively presumably due to chronic rejection. One patient required a lower doses of exogenous insulin after a severe systemic herpes virus infection.

The actual survival rates at 1 year for patients, pancreatic and renal grafts was $94 \%, 72 \%$ and $93 \%$ respectively. After a mean observation time of 57 months, 9 of 16 (57\%) pancreas transplants and 11 of $15(73 \%)$ renal transplants are currently functioning (Fig. 1).

Creatinin levels at 1 and 2 years were $1,4(0,7-2,4) \mathrm{mg} \%$ and $1,5(1,1-2,3) \mathrm{mg} \%$ respectively.

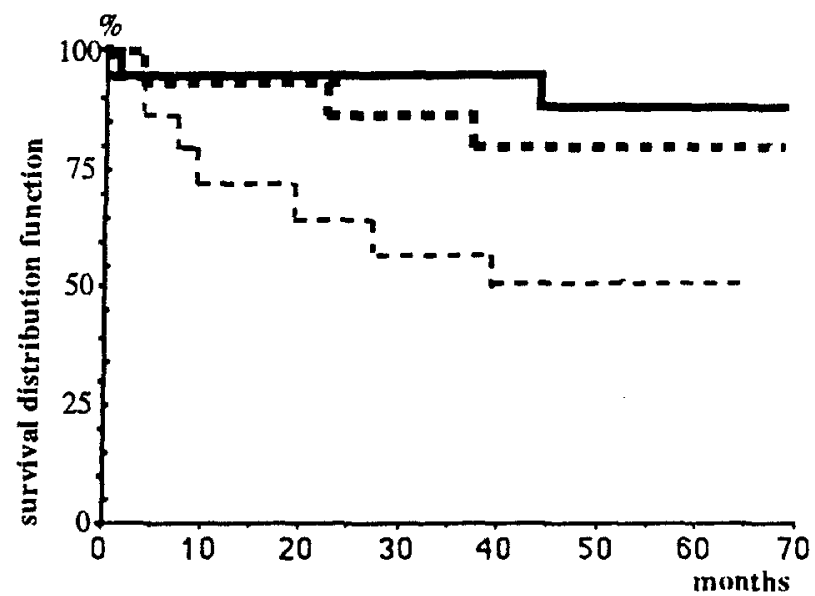

Fig. 1. Patient survival $(-)$ and graft survival analysis after combined kidney $(-\rightarrow)$ and pancreas (- - ) transplantation with the delayed duct occlusion technique

Surgical complications occurred in seven patients, superficial wound infection as well as fistula formation most frequently. Two patients required reoperation for haemorrhage at the graft site. Apart from the patient who died of progressive macroangiopathy 4 years following transplantation, all patients with functioning grafts are fully rehabilitated with normal carbohydrate metabolism (Fig. 2). 
S6

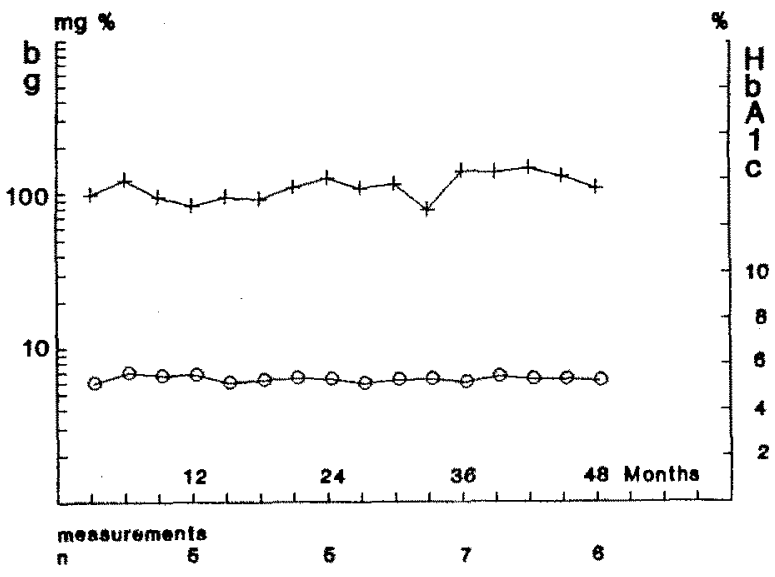

Fig. 2. Long - term glycaemic profiles in patients with successful pancreas transplantation using the delayed duct occlusion technique $++=$ blood glucose; $a-0=$ HbAlc

\section{Bladder drainage}

Two and 4 weeks after combined kidney pancreas transplantation two patients died of bacterial pneumonia, which occurred after high-dose steroid treatment for acute rejection. One patient succumbed to cytomegalovirus pneumonia and one was lost to a cerebral haemorrhage 6 days after transplantation. Two patients ( 24 and 41 years old) died of myocardial infarction with normally functioning grafts 3 months and 8 months respectively after transplantation. A total of seven pancreas transplants were lost due to rejection between 1 week and 6 months after transplantation. Venous thrombosis was the reason for graft loss in three cases, and in one case necrotizing pancreatitis led to the graft loss. Two patients required relaparotomy for bleeding complications and two patients, for leakage at the pancreatocystostomy. Survival at 1 year was $90 \%, 74 \%$ and $89 \%$ for patients, pancreatic and renal grafts. After a mean observation time of 24 months, 32 of $38(84.2 \%)$ patients are currently alive. As of 31 December $1990,64.4 \%$ of the pancreas and $74.7 \%$ of the renal transplants are functioning (Fig. 3).

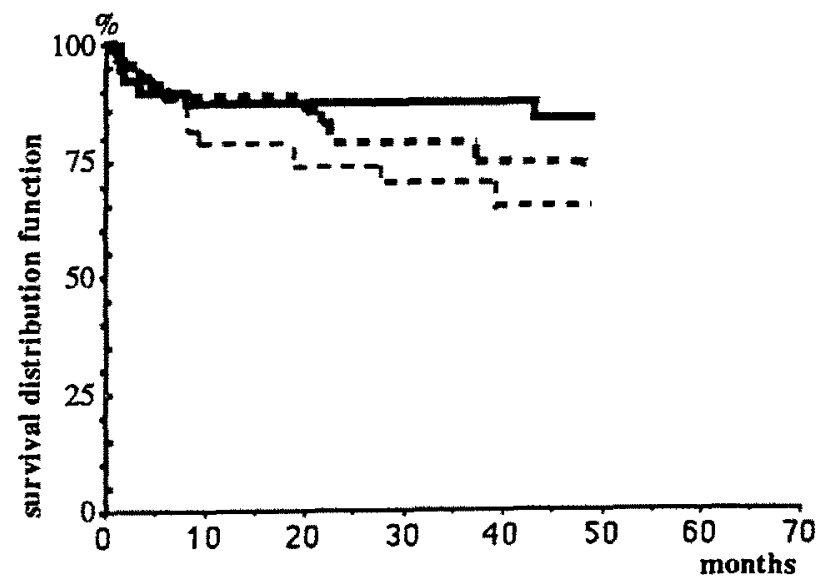

Fig. 3. Patient survival (-) and graft survival analysis after combined kidney $(--)$ and pancreas $\left(-_{-}\right)$transplantation with bladder drainage technique
A. Königsrainer et al.: Pancreas transplantation - long-term results

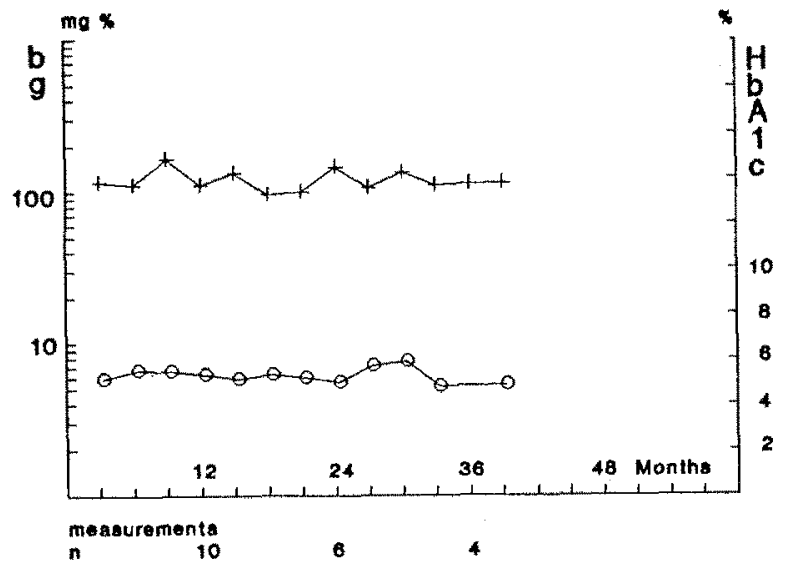

Fig. 4. Long - term glyceamic profiles in patients with successful pancreas transplantation using the bladder drainage technique $\leftarrow=$ blood glucose; $0-0=$ HbAlc

Of the 32 patients still alive, $75 \%$ are free of exogenous insulin and $85.7 \%$ have a functioning renal transplant. Creatinin levels at 1 and 2 years were $1,3(0,8-2,1) \mathrm{mg} \%$ and $1,2(1,0-1,5) \mathrm{mg} \%$. In these patients, only seven episodes of urinary tract infection were observed, which were easily controlled with antibiotics. The metabolic control of these patients is depicted in Figure 4.

\section{Discussion}

In contrast to other solid organs, the surgical technique of pancreas transplantation is still not standardized. Only the intraperitoneal positioning of the graft seems to be generally accepted as the best site. Although most centres now use the whole organ, we still transplant a segment which we call subtotal, since it also comprises major parts of the head of the pancreas. One of its advantages is that it does not interfere with the vasculature of the liver graft even in case of anomalies of the hepatic artery. The low incidence of urinary tract infection is most likely due to the fact that no duodenal segment is used. Furthermore, pancreatic enzymes are not activated and therefore complications such as urethritis and balanitis (Tom et al. 1987; Roxvall et al. 1988) have never been observed in our series. Other complications associated with the transplantation of a segment of the duodenum together with the pancreas, such as bleeding or perforation, are avoided and transcystoscopical biopsies can easily be performed under direct vision. Drainage of pancreas secretion without the duodenum seems to not have any detrimental influence on the bladder mucosa. We have performed serial biopsies up to 3 years following transplantation. No lesions have been detected in any of these biopsies (Königsrainer et al. 1990c). Since carbohydrate metabolism is similarly well controlled, as compared to total pancreas allografts the number of islets transplanted with such a reduced-size graft appears to be sufficient.

After transplantation of a pancreatic segment, a higher incidence of venous thrombosis was observed, as compared to transplantation of the entire pancreas. With regard to thrombotic complications, no firm conclusions 
can be drawn from our series since the number of transplants is still rather small. It is interesting to note, however, that no venous thrombosis occurred after delayed duct occlusion in comparison to three such surgical complications after bladder drainage. We believe, however, that two of these events can be ascribed to insufficient surgical technique, and one thrombosis occurred in a graft which had been improperly perfused. The duct occlusion technique first described by our group provides two major advantages. It prevents further damage during the immediate post-operative period thanks to the occlusion procedure and facilitates precise monitoring of the transplant's exocrine function.

Since pancreatic secretion can also be easily obtained after bladder drainage by inserting a catheter into the pancreatic duct (the catheter can be simply removed when it is no longer required), we currently give preference to this method. Because of the severe complications caused by the duodenal segment we prefer subtotal rather than whole pancreas transplantation.

\section{References}

Dubemard JM, Traeger J, Neyra P, Touraine JL, Tronchant D, Blanc Bunnot $N$ (1978) A new method of preparation of segmental pancreatic graft for transplantation: trials in dogs and in men. Surgery 84: 633-639

Klima G, Margreiter R, Königsrainer A, Steiner E, Schmid T (1989) Pancreatic juice cytology (PJC) for early detection of pancreas allograft rejection. Transplant Proc 21: 2782-2783

Königsrainer A, Steiner E, Spielberger M, Aigner F, Schmid T, Margreiter R (1987) Delayed duct occlusion: a new technique for pancreas transplantation. Lang Arch Chir 372: 67-72
Königsrainer A, Schmid Th, Habringer Ch, Then P, Margreiter R (1990a) A new technique for venous anastomosis of pancreatic allografts. Eur Surg Res 22: 279-282

Königsrainer A, Reibnegger G, Öfner D, Klima G, Tauscher T, Margreiter R (1990b) Pancreatic juice Neopterin excretion reliable marker for detection of pancreatic allograft rejection.Transplant Proc 22: 671-672

Königsrainer A, Feichtinger H, Waitz W, Ebner A, Scheiber K, Mack D, Margreiter R (1990c) Does pancreatic juice have a detrimental effect on bladder mucosa? Transplant Proc 22: 16001601

Margreiter R, Königsrainer A, Steiner E, Schmid T, Pernthaler H (1988) Improved results of pancreatic transplantation with the delayed duct occlusion technique.Transplant Proc 19: 889-890

Margreiter R, Steiner E, Königsrainer A, Aigner F, Spielberger M, Bösmüller C (1989) Results of pancreas transplantation in Innsbruck. In: Dubernard JM, Sutherland DER (eds) Intemational handbook of pancreas transplantation, Kluwer Academic, Dordrecht, pp 379-381

Roxvall LI, Frisk B, Hedman L, Nyberg G (1988) Graft pancreatitis and hemorrhagic cystitis. Transplant Int 1: 228-229

Sollinger HW, Kalayoglu M, Hoffmann RM, Deierhoi MH, Belzer FO (1985) Experience with pancreaticocystostomy in 24 consecutive pancreas transplants. Transplant Proc 17: 141-143

Steiner E, Klima G, Niederwieser D, Königsrainer A, Margreiter R (1987) Monitoring of the pancreatic allograft by analysis of exocrine secretion. Transplant Proc19: 2336-2338

Sutherland DER, Moudry KC (1989) Pancreas transplantation registry report. Transplant Proc 21: 2759-2762

Tom WW, Munda R, First MR, Alexander JW (1987) Autodigestion of the glans penis and urethra by activated pancreatic exocrine enzymes. Surgery 102: 99-101

Dr. Alfred Königsrainer

Dep. Transplant Surgery - University Hospital

Anichstrasse 35

A - 6020 INNSBR U CK 